University of New Mexico

UNM Digital Repository

Mathematics and Statistics Faculty and Staff

Publications

Academic Department Resources

8-2020

The Polar form of a Neutrosophic Complex Number

Florentin Smarandache

Riad K. Al-Hamido

Mayas Ismail

Follow this and additional works at: https://digitalrepository.unm.edu/math_fsp

Part of the Logic and Foundations Commons, Other Mathematics Commons, and the Set Theory Commons 


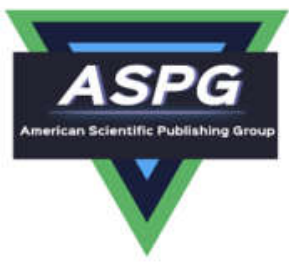

\title{
The Polar form of a Neutrosophic Complex Number
}

\author{
Riad K. Al-Hamido ${ }^{1}$, Mayas Ismail2*, Florentin Smarandache ${ }^{3}$ \\ ${ }^{1}$ Department of Mathematics, College of Science, AlFurat University, Deir-ez-Zor, Syria. \\ ${ }^{1}$ E-mail: riad-hamido1983@hotmail.com \\ ${ }^{2 *}$ Department of Computer Engineering, International University for Science and Technology, Daraa, Syria. \\ ${ }^{2 *}$ E-mail: mayas.n.ismail@gmail.com; Tel.: (+963937082244) \\ ${ }^{3}$ Department of Mathematics, University of New Mexico, Gallup, NM 87301, USA. \\ 3E-mail: smarand@unm.edu
}

\begin{abstract}
In this paper, we will define the exponential form of a neutrosophic complex number. We have proven some characteristics and theories, including the conjugate of the exponential form of a neutrosophic complex number, division of the exponential form of a neutrosophic complex numbers, multiplication of the exponential form of a neutrosophic complex numbers. In addition, we have given the method of changing from the exponential to the algebraic form of a complex number.
\end{abstract}

Keywords: Neutrosophic numbers, neutrosophic complex number, the exponential form of a neutrosophic complex number.

\section{Introduction}

The American scientist and philosopher F. Smarandache came to place the neutrosophic logic in [1-5], and this logic is as a generalization of the fuzzy logic [6], conceived by L. Zadeh in 1965.

The neutrosophic logic is of great importance in many areas of them, including applications in image processing [78], the field of geographic information systems [9], and possible applications to database [10-11], and have applications in the medical field [12-15], and in neutrosophic bitopology in [16-18], and in neutrosophic algebra in [19-23], professor F. Smarandache presented the definition of the standard form of neutrosophic real number and conditions for the division of two neutrosophic real numbers to exist, he defined the standard form of neutrosophic complex number [24], and Y. Alhasan presented the properties of the concept of neutrosophic complex numbers including the conjugate of neutrosophic complex number, division of neutrosophic complex numbers, the inverted neutrosophic complex number and the absolute value of a neutrosophic complex number and theories related to the conjugate of neutrosophic complex numbers, and that the product of a neutrosophic complex number by its conjugate equals the absolute value of number [25]. 
This paper aims to study and define the exponential form of a neutrosophic complex number by defining the conjugate of the exponential form of a neutrosophic complex number, division of the exponential form of the neutrosophic complex numbers, and multiplication of the exponential form of a neutrosophic complex numbers.

\section{Preliminaries}

In this section, we present the basic definitions that are useful in this research.

\section{Definition 2.1 [24]}

A neutrosophic number has the standard form:

$$
\mathrm{a}+\mathrm{b} I
$$

where $\mathrm{a}, \mathrm{b}$ are real or complex coefficients, and $I=$ indeterminacy, such $0 . I=0$

$I^{n}=I$ for all positive integer $\mathrm{n}$.

If the coefficients $\mathrm{a}$ and $\mathrm{b}$ are real, and then $\mathrm{a}+\mathrm{b} I$ is called neutrosophic real number.

For example: $5+7 I$

\section{Definition $2.2[25]$}

$\mathrm{z}$ is a neutrosophic complex number, if it takes the following standard form:

$$
\mathrm{z}=\mathrm{a}+\mathrm{b} I+\mathrm{ci}+\mathrm{di} I
$$

Where a, b, c, d are real coefficients, and $I=$ indeterminacy, and $i^{2}=-1$.

\section{Division of Neutrosophic Real Numbers [24]}

$$
\left(a_{1}+b_{1} I\right) \div\left(a_{2}+b_{2} I\right)=?
$$

We denote the result by:

$$
\begin{gathered}
\frac{a_{1}+b_{1} \mathrm{I}}{a_{2}+b_{2} \mathrm{I}}=\mathrm{x}+\mathrm{yI} \\
x=\frac{a_{1}}{a_{2}}
\end{gathered}
$$

and

$$
y=\frac{a_{2} b_{1}-a_{1} b_{2}}{a_{2}\left(a_{2}+b_{2}\right)}
$$

Definition 2.4 [25] 
Suppose that $\mathrm{z}=\mathrm{a}+\mathrm{b} I+\mathrm{ci}+\mathrm{di} I$ is a neutrosophic complex number, then the absolute value of a neutrosophic complex number defined by the following form:

$$
|z|=\sqrt{(a+b I)^{2}+(c+d I)^{2}}
$$

\section{The Polar form of a Neutrosophic Complex Number}

In this section, we present and study the exponential form of a neutrosophic complex number.

\section{Definition 3.1}

We define the Exponential Form of a Neutrosophic Complex Number as follows:

$$
z=r e^{i(\theta+I)}
$$

where $r$ the Absolute Value of the neutrosophic complex number.

\section{Remark 3.1.1:}

From the general form:

$$
\begin{gathered}
\mathrm{z}=\mathrm{a}+\mathrm{b} I+\mathrm{ci}+\mathrm{di} I \\
z=r\left(\frac{\mathrm{a}+\mathrm{b} I}{r}+\frac{\mathrm{ci}+\mathrm{di} I}{r}\right) \\
z=r\left(\frac{\mathrm{a}+\mathrm{b} I}{r}+i \cdot \frac{\mathrm{c}+\mathrm{d} I}{r}\right)
\end{gathered}
$$

Remark 3.1.2:

$$
r=|z|=\sqrt{(a+b I)^{2}+(c+d I)^{2}}
$$




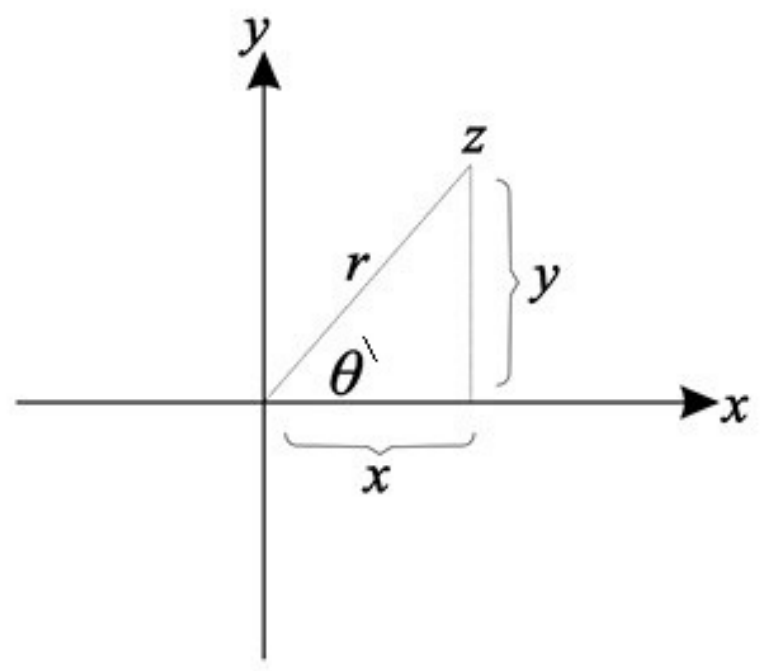

Figure 1: The Geometrical Figure

The formula neutrosophically works in the following way:

$\mathrm{x}=\mathrm{a}+\mathrm{bI}$ is a neutrosophic number whose determinate part is "a" and indeterminate part is "bI", where $\mathrm{I}=$ indeterminacy;

similarly $\mathrm{y}=\mathrm{c}+\mathrm{dI}$ is a neutrosophic number whose determinate part is "c" and indeterminate part is "dI";

$\Theta=\theta+\mathrm{I}$ is a neutroosphic angle, whose determinate part is $\Theta$ ("theta") and indeterminate part is "I".

It is a big "Theta" $\Theta$ (inside the geometrical figure) and small "theta" $\theta$ in the formulas.

That means that we work with two lengths $\mathrm{x}$ and $\mathrm{y}$ that are not well-known (they were approximated), and an angle $\Theta$ (Theta) that is not well known either (it was approximated by $\theta$ plus some indeterminacy I).

$$
\begin{gathered}
\cos (\theta+I)=\frac{x}{r}=\frac{\mathrm{a}+\mathrm{b} I}{r}, \quad \sin (\theta+I)=\frac{y}{r}=\frac{\mathrm{c}+\mathrm{d} I}{r} \\
z=r(\cos (\theta+I)+i \cdot \sin (\theta+I))
\end{gathered}
$$

Exponential Form:

$$
z=r e^{i(\theta+I)}
$$

\section{Definition 3.2}

Trigonometric formula

$$
z=r(\cos (\theta+I)+i \sin (\theta+I))
$$




\section{Properties}

In this section, we present some important properties of the exponential form.

\section{Multiplying the exponential forms of the neutrosophic complex numbers}

Suppose that $z_{1}, z_{2}$ are two neutrosophic complex numbers, where

$$
z_{1}=r_{1} e^{i\left(\theta_{1}+I_{1}\right)} \text { and } z_{2}=r_{2} e^{i\left(\theta_{2}+I_{2}\right)}
$$

If $I_{1}+I_{2}=I$

Definition 4.1

$$
z_{1} \cdot z_{2}=r_{1} r_{2} e^{i\left(\theta_{1}+\theta_{2}+I\right)}
$$

\section{Remark 4.1.1:}

$$
\begin{gathered}
z_{1} \cdot z_{2}=r_{1} e^{i\left(\theta_{1}+I_{1}\right)} \cdot r_{2} e^{i\left(\theta_{2}+I_{2}\right)} \\
z_{1} \cdot z_{2}=r_{1} r_{2}\left(e^{i\left(\theta_{1}+I_{1}\right)} \cdot e^{i\left(\theta_{2}+I_{2}\right)}\right) \\
z_{1} \cdot z_{2}=r_{1} r_{2} e^{i\left(\theta_{1}+\theta_{2}+I_{1}+I_{2}\right)}
\end{gathered}
$$

$I_{1}+I_{2}=I$

Then

$$
z_{1} \cdot z_{2}=r_{1} r_{2} e^{i\left(\theta_{1}+\theta_{2}+I\right)}
$$

\section{Example 4.1.2}

$$
\begin{aligned}
& \text { If } z_{1}=r_{1} e^{i\left(\frac{\pi}{4}+I\right)} \text { and } z_{2}=r_{2} e^{i\left(\frac{3 \pi}{4}+I\right)} \\
& \qquad z_{1} \cdot z_{2}=r_{1} r_{2} e^{i\left(\frac{\pi}{4}+3 \frac{\pi}{4}+I\right)}=r_{1} r_{2} e^{i(\pi+I)}
\end{aligned}
$$

\section{Division of the exponential forms of neutrosophic complex numbers}

Suppose that $z_{1}, z_{2}$ are two neutrosophic complex numbers, where

$$
z_{1}=r_{1} e^{i\left(\theta_{1}+I_{1}\right)} \text { and } z_{2}=r_{2} e^{i\left(\theta_{2}+I_{2}\right)}
$$

If $I_{1}-I_{2}=I$

then

\section{Definition 4.2}




$$
\frac{z_{1}}{z_{2}}=\frac{r_{1}}{r_{2}} \boldsymbol{e}^{i\left(\theta_{1}-\theta_{2}+I\right)}
$$

\section{Remark 4.2.1:}

Depending on [25]

$$
z \cdot \bar{z}=|z|^{2}=r^{2}
$$

When $\mathrm{r}=1$ we get $\Rightarrow$

$$
\bar{z}=\frac{1}{Z}=\frac{e^{0}}{e^{i(\theta+I)}}=e^{-i(\theta+I)}
$$

Then

$$
\begin{gathered}
\frac{z_{1}}{z_{2}}=\frac{r_{1} e^{i\left(\theta_{1}+I_{1}\right)}}{r_{2} e^{i\left(\theta_{2}+I_{2}\right)}} \\
\frac{z_{1}}{z_{2}}=\frac{r_{1}}{r_{2}}\left(\frac{e^{i\left(\theta_{1}+I_{1}\right)}}{e^{i\left(\theta_{2}+I_{2}\right)}}\right) \\
\frac{z_{1}}{z_{2}}=\frac{r_{1}}{r_{2}}\left(e^{i\left(\theta_{1}+I_{1}\right)} \cdot \frac{1}{e^{i\left(\theta_{2}+I_{2}\right)}}\right) \\
\frac{z_{1}}{z_{2}}=\frac{r_{1}}{r_{2}}\left(e^{i\left(\theta_{1}+I_{1}\right)} \cdot e^{-i\left(\theta_{2}+I_{2}\right)}\right)
\end{gathered}
$$

$I_{1}-I_{2}=I$

Then

$$
\frac{z_{1}}{z_{2}}=\frac{r_{1}}{r_{2}} \boldsymbol{e}^{i\left(\theta_{1}-\theta_{2}+I\right)}
$$

\section{Example 4.2.2}

If $z_{1}=r_{1} e^{i\left(\frac{\pi}{4}+I\right)}$ and $z_{2}=r_{2} e^{i\left(\frac{3 \pi}{4}+I\right)}$

$$
\frac{z_{1}}{z_{2}}=\frac{r_{1}}{r_{2}} e^{i\left(\frac{\pi}{4}-3 \frac{\pi}{4}+I\right)}=\frac{r_{1}}{r_{2}} e^{-i\left(\frac{\pi}{2}+I\right)}
$$

\section{The conjugate of the exponential form of a neutrosophic complex numbers 4.3}

Suppose that $\mathrm{z}$ is a neutrosophic complex number, where 


$$
z=r e^{i(\theta+I)}
$$

We denote the conjugate of a neutrosophic complex number by $\bar{z}$ and define it by the following form:

$$
\bar{z}=r e^{-i(\theta+I)}
$$

\section{Example 4.3.1}

$$
\begin{aligned}
& z=r e^{i\left(\frac{\pi}{2}+I\right)} \\
& \bar{z}=r e^{-i\left(\frac{\pi}{2}+I\right)}
\end{aligned}
$$

\section{Remark 4.4}

If $I=0$ we will return to the basic formula for the complex number.

$$
\begin{gathered}
z=r e^{i(\theta+0)} \\
z=r e^{i(\theta)}
\end{gathered}
$$

\section{Conclusion}

In this paper, we defined the exponential form of a neutrosophic complex number and demonstrated this with appropriate proof, and many examples were presented to illustrate the concepts introduced in this paper.

\section{Future Research Directions}

As a future work, some special cases related to exponential form can be discussed and benefit from this article in many engineering sciences, including theories of control and signal processing.

\section{References}

[1] F. Smarandache,"Neutrosophic Precalculus and Neutrosophic Calculus", EuropaNova asbl, Clos du Parnasse, 3E 1000, Bruxelles, Belgium, 2015.

[2] F. Smarandache, "An Introduction to Neutrosophic Probability Applied in Quantum Physics", Bulletin of the American Physical Society, 2009 APS April Meeting, Volume 54, Number 4, Saturday-Tuesday, May 2-5, 2009; Denver, Colorado, USA, http://meetings.aps.org/Meeting/APR09/Event/102001.

[3] F. Smarandache, "An Introduction to Neutrosophic Probability Applied in Quantum Physics", SAO/NASA ADS Physics Abstract Service, http://adsabs.harvard.edu/abs/2009APS..APR.E1078S. 
[4] F. Smarandache, "A Unifying Field in Logics: Neutrosophic Logic, Neutrosophic Set", Neutrosophic Probability and Statistics, (1999), (2000), (2003), (2005).

[5] F. Smarandache; Huda E.Khalid,"Neutrosophic Precalculus and Neutrosophic Calculus", Second enlarged edition, Pons asbl, 5, Quai du Batelage, Brussels, Belgium, European Union, 2018.

[6] L. Zadeh, "Fuzzy sets, Inform and Control", 8, pp.338-353, 1965.

[7] A. A Salama; I. M Hanafy; Hewayda Elghawalby Dabash M.S,"Neutrosophic Crisp Closed Region and Neutrosophic Crisp Continuous Functions", New Trends in Neutrosophic Theory and Applications.

[8] A. A Salama; Hewayda Elghawalby; M.S. Dabash; A. M. NASR ,"Retrac Neutrosophic Crisp System For Gray Scale Image", Asian Journal of Mathematics and Computer Research, Vol. 24, 104-117-22, 2018.

[9] A. A Salama,"Basic Structure of Some Classes of Neutrosophic Crisp Nearly Open Sets \& Possible Application to GIS Topology", Neutrosophic Sets and Systems, Vol. 7, 18-22, 2015.

[10] A. A Salama, F. Smarandache," Neutrosophic Crisp Set Theory", Neutrosophic Sets and Systems, Vol. 5, 1-9, 2014.

[11] F. Smarandache, "Neutrosophy and Neutrosophic Logic, First International Conference on Neutrosophy, Neutrosophic Logic, Set, Probability, and Statistics", University of New Mexico, Gallup, NM 87301, USA ,2002.

[12] M. Abdel-Basset; M. Mai, E. Mohamed; C. Francisco;H. Z. Abd El-Nasser, "Cosine similarity measures of bipolar neutrosophic set for diagnosis of bipolar disorder diseases", Artificial Intelligence in Medicine Vol. 101 , 101735,2019.

[13] M. Abdel-Basset; E. Mohamed; G. Abduallah; and S. Florentin, "A novel model for evaluation Hospital medical care systems based on plithogenic sets", Artificial intelligence in medicine 100, 101710, 2019.

[14] M. Abdel-Basset; G. Gunasekaran Mohamed; G. Abduallah. C. Victor,"A Novel Intelligent Medical Decision Support Model Based on Soft Computing and IoT", IEEE Internet of Things Journal, Vol. 7, 2019.

[15] M. Abdel-Basset; G. Abduallah; G. Gunasekaran; L. Hoang Viet, "A novel group decision making model based on neutrosophic sets for heart disease diagnosis", Multimedia Tools and Applications, pp.1-26, 2019.

[16] R.K. Al-Hamido,"Neutrosophic Crisp Supra Bi-Topological Spaces", International Journal of Neutrosophic Science, Vol. 1, 66-73, 2018.

[17] R.K. Al-Hamido,"Neutrosophic Crisp Bi-Topological Spaces", Neutrosophic Sets and Systems, vol. 21, pp.66$73,2018$.

[18] R.K. Al-Hamido; T. Gharibah; S. Jafari, F.Smarandache, "On Neutrosophic Crisp Topology via N-Topology", Neutrosophic Sets and Systems, vol. 21, 96-109, 2018.

[19] V. Christianto; F. Smarandache; M. Aslam, "How we can extend the standard deviation notion with neutrosophic interval and quadruple neutrosophic numbers", International Journal of Neutrosophic Science, vol. 2, No. 2, pp.72-76, 2020.

[20] E. O. Adeleke; A. A. A. Agboola; F. Smarandache, "Refined Neutrosophic Rings I", International Journal of Neutrosophic Science, vol. 2 , No. 2, pp.77-81, 2020.

[21] E. O. Adeleke; A. A. A. Agboola; F. Smarandache, "Refined Neutrosophic Rings II", International Journal of Neutrosophic Science, vol 2. , No. 2, pp.89-94, 2020.

[22] M. A. Ibrahim; A. A. A. Agboola; E. O. Adeleke; S. A. Akinleye, "Introduction to Neutrosophic Subtraction Algebra and Neutrosophic Subtraction Semigroup", International Journal of Neutrosophic Science, vol. 2 , No. 1, pp.47-62, 2020.

[23] A. Hatip,"The Special Neutrosophic Functions", International Journal of Neutrosophic Science, vol. 4 , No. 2 , pp.104-116, 2020.

[24] F. Smarandache, "Introduction to Neutrosophic Statistics", Sitech-Education Publisher, pp.34-44, 2014.

[25] Y. Alhasan, "Concepts of Neutrosophic Complex Numbers ", International Journal of Neutrosophic Science, Vol. 8 No. 1, pp. 9-18, 2020.

[26] Samah Ibrahim Abdel Aal, Mahmoud M. A. Abd Ellatif, Mohamed Monir Hassan: Two Ranking Methods of Single Valued Triangular Neutrosophic Numbers to Rank and Evaluate Information Systems Quality, Neutrosophic Sets and Systems, vol. 19,pp. 132-141, 2018. http://doi.org/10.5281/zenodo.1235339 
[27] Faruk Karaaslan: Gaussian single-valued neutrosophic numbers and its application in multi-attribute decision making, Neutrosophic Sets and Systems, vol. 22, pp. 101-117, 2018. DOI: 10.5281/zenodo.2159762

[28] Surapati Pramanik, Rama Mallick: VIKOR based MAGDM Strategy with Trapezoidal Neutrosophic Numbers, Neutrosophic Sets and Systems, vol. 22, pp. 118-130,2018. DOI: 10.5281/zenodo.2160840

[29] Irfan Deli: Operators on Single Valued Trapezoidal Neutrosophic Numbers and SVTN-Group Decision Making, Neutrosophic Sets and Systems, vol. 22, pp. 131-150, 2018. DOI: 10.5281/zenodo.2160120

[30] Bibhas C. Giri, Mahatab Uddin Molla, Pranab Biswas: TOPSIS Method for MADM based on Interval Trapezoidal Neutrosophic Number, Neutrosophic Sets and Systems, vol. 22, pp. 151-167, 2018.DOI: 10.5281/zenodo.2160749 